\title{
THE f-NORM; A GENERALIZATION OF THE NORM OF FUNCTIONAL ANALYSIS
}

\author{
Norris Sookoo \\ University of Trinidad and Tobago, San Fernando, Trinidad and Tobago
}

\begin{abstract}
A generalisation, $f$-norm, of the concept of norm as defined in Functional Analysis is introduced. The norm of $\lambda x$ is $|\lambda|$ times the norm of $x$. In the definiton of the $f$-norm, this requirement is relaxed, so that the $f$-norm of $\lambda x$ is $f(|\lambda|)$ times the $f$-norm of $x$, where $f$ is a function satisfying certain properties, which are presented. Examples of $f$-norms are given, the metric induced by an $f$-norm is considered and results concerning continuity of certain functions obtained. Sets that are convex with respect to an $f$-norm are studied. Limits with respect to the $f$-norm are also considered.
\end{abstract}

\section{Indexing terms/Keywords}

$f$-norm; metric; continuity; convexity

\section{Academic Discipline And Sub-Disciplines}

Mathematics

\section{SUBJECT CLASSIFICATION}

Mathematics Subject Classification 46E99; American Mathematical Society Classification

\section{TYPE (METHOD/APPROACH)}

Provide examples of relevant research types, methods, and approaches for this field: Generalization

\section{Council for Innovative Research}

Peer Review Research Publishing System

Journal: JOURNAL OF ADVANCES IN MATHEMATICS

Vol .11, No.7

www.cirjam.com, editorjam@gmail.com 


\section{INTRODUCTION}

The concept of norm is fundamental in Mathematics and various types of norm are used according to the requirements in different areas. It is well known that norms have been used extensively in the study of functions. New types of norm continue to be introduced periodically. Urbanski [7] gave a generalization of the Koshi-Shimogaki and Amemiya's norm defined in a modular space over a field with valuation. Lee and Verleysen [2] considered a generalization of the $L_{p}$ norm suitable for time-series data with particular regard for the temporal structure. Ramsey and Silverman [4] and Roni, Delannay, Conan-Guez and Verleysen [5] also took the temporal structure of data into account. Hatami [1] studied generalizations of Gower's norm, which are defined in terms of integrals. He obtained conditions on the structure of integrals which corresponds to norm functions. Matkowski [3] gave some results about a functional which becomes the $L^{p}-$ norm under certain conditions.

One of the conditions that a norm must satisfy is that the norm of $\lambda x$ be equal to $|\lambda|\|x\|$. In this paper, we introduce a generalization of the concept of norm, namely, the $f$-norm, which eliminates the strict requirement that the norm of $\lambda x$ be equal to the modulus of lambda times the norm of $\mathrm{x}$. We require instead that the norm of $\lambda x$ be equal to a function $f$ of lambda times the norm of $\mathrm{x}$. We present some properties of this function, provide examples of $f$-norms, consider the metric introduced by an $f$-norm and obtain results concerning continuity of certain functions. Many of the results presented in this paper are generalizations of known results about norms. In some cases, the proofs in this paper are quite similar to proofs of corresponding results about norms, but in other cases, the proofs presented here are significantly different. Basically, our purpose is to consider how results that are given with respect to the usual norm may be modified when the norm is replaced by the $f$-norm.

\section{Definitions and Notations}

Notation 2.1. $\mathbb{C}$ represents the set of complex numbers and $\mathfrak{R}$ represents the set of real numbers.

Definition 2.2. An $f$-norm on a real or complex linear space $\aleph$ is a non-negative real-valued function $\eta$ on $\aleph$ with the following properties:

$$
\begin{aligned}
& \eta(x)=0 \Leftrightarrow x=0, \forall x \in \aleph \\
& \eta(\lambda x)=f(|\lambda|) \eta(x), \forall \lambda \in R \text { and } x \in \aleph \\
& \eta(x+y) \leq \eta(x)+\eta(y), \forall x, y \in \aleph
\end{aligned}
$$

where $f$ is a continuous, real-valued monotone-increasing function on the non-negative real numbers such that the gradient of $f$ is positive and non-increasing.

Notation 2.3. (c.f. page 54, III, Simmons [6]). Let $\eta$ be an $f$-norm on a space $\aleph$, and let

$$
\rho_{\eta}(x, y)=\eta(x-y), \forall x, y \in \mathfrak{K}
$$

Definition 2.4. (c.f. page 24, Yosida [8]) Let $\eta(x)$ be an $f$-norm on $X, c$ be any positive number and

$$
M_{n c}=\{x \in X \mid \eta(x) \leq n c\}, n \in \mathfrak{R}, n \geq 1 \text {. }
$$

(i) $M_{c}$ is called convex if $x, y \in M_{c}$ and $0<\alpha<1 \Rightarrow \alpha x+(1-\alpha) y \in M_{c}$

(ii) $M_{c}$ is called $k$-convex if $x, y \in M_{c}$ and $0<\alpha<1 \Rightarrow \alpha x+(1-\alpha) y \in M_{k c}$

(iii) $M_{c}$ is called balanced if $x \in M_{c}$ and $|\alpha| \leq 1 \Rightarrow \alpha x \in M_{c}$

(iv) $M_{c}$ is called absorbing if for any $x \in X, \exists \alpha>0$ э $\alpha^{-1} x \in M_{c}$.

Notation 2.5. (c.f. page 30, Yosida [8]) Given an $f$-norm $\eta$ and the induced metric $\rho_{\eta}$, the convergence $\lim _{n \rightarrow \infty} \rho_{\eta}\left(x_{n}, x\right)=0$ on an $f$-normed space $X$ will be denoted by $\rho_{\eta}-\lim _{n \rightarrow \infty} x_{n}=x$ and we say that $\left\{x_{n}\right\}$ converges strongly to $x$. 


\section{Properties of the function $f$}

Theorem 3.1. If $\eta$ is an $f$ norm with respect to a function $f$, then $f$ must satisfy the condition

$$
f(\alpha+\beta) \leq f(\alpha)+f(\beta), \forall \alpha, \beta \in R \text { э } \alpha, \beta \geq 0
$$

Proof.

$\eta(\alpha x+\beta x) \leq \eta(\alpha x)+\eta(\beta x), \forall x \in \aleph$ э $x \neq 0$ and $\forall \alpha, \beta \in \mathrm{R}$ э $\alpha \geq 0$, and $\beta \geq 0$.

Therefore $\quad f(\alpha+\beta) \eta(x) \leq f(\alpha) \eta(x)+f(\beta) \eta(x)$

Therefore $\quad f(\alpha+\beta) \leq f(\alpha)+f(\beta)$

Theorem 3.2. $f(0)=0$

Proof. For $x \in \aleph$ э $x \neq 0, \eta(0 x)=f(|0|) \eta(x)$

Therefore $\quad f(0) \eta(x)=0$

Therefore $\quad f(0)=0$

Theorem 3.3. $f(1)=1$

Proof. For $x \neq 0, \eta(1 x)=\eta(x)$

Therefore $\quad f(1) \eta(x)=\eta(x)$

Therefore $\quad f(1)=1$

Theorem 3.4. $f(a) \leq a, \forall a \in \mathfrak{R} \ni a>1$.

Proof. Suppose that $f(b)>b$ for some $b \in \mathfrak{R} \ni b>1$. Let $A$ be the point $(1,1)$ and $B$ be the point $(b, f(b))$.

Then gradient of $A B=\frac{f(b)-1}{b-1}>\frac{b-1}{b-1}=1$.

Hence there exists some value $c \ni 1 \leq c \leq b$, and the gradient of the graph of $y=f(x)$ at the point $(c, f(c))=$ gradient of $A B>1$.

Hence the gradient of this graph $>1$ for all point of $[0,1]$, and so $(1,1)$ cannot be on the graph. However, we know that $(1,1)$ is on the graph and hence $f(a) \leq a, \forall a>1$.

\section{The metric induced by the $f$-norm}

Theorem $4.1 \eta\left(x_{1}-x_{2}\right) \geq\left|\eta\left(x_{1}\right)-\eta\left(x_{2}\right)\right|$ (cf Proposition 1, page 23 Yosida [8])

Proof. $\quad \eta\left(x_{1}-x_{2}\right)+\eta\left(x_{2}\right) \geq \eta\left[\left(x_{1}-x_{2}\right)+x_{2}\right]=\eta\left(x_{1}\right)$

Therefore $\quad \eta\left(x_{1}-x_{2}\right) \geq \eta\left(x_{1}\right)-\eta\left(x_{2}\right)$ (I)

From Theorem $3.3 \eta\left(x_{1}-x_{2}\right)=\eta\left[(-1)\left(x_{2}-x_{1}\right)\right]=f(|-1|) \eta\left(x_{2}-x_{1}\right)$

$$
=\eta\left(x_{2}-x_{1}\right) \geq \eta\left(x_{2}\right)-\eta\left(x_{1}\right) \quad \text { from (I) }
$$

Therefore

$$
\eta\left(x_{1}-x_{2}\right) \geq\left|\eta\left(x_{1}\right)-\eta\left(x_{2}\right)\right|
$$

The following corollary follows immediately.(cf page 212, Simmons [6])

Corollary 4.2. $\eta$ is continuous. 
The following theorem is a generalization of a well-known result relating metrics to norms, (cf Simmons [6] page 54)

Theorem 4.3. $\rho_{\eta}$ is a metric on $\aleph$.

Proof. $\rho_{\eta}$ satisfies the conditions to be a metric as shown below.

$$
\rho_{\eta}(x, y)=\eta(x-y) \geq 0
$$

Also

$$
\rho_{\eta}(x, y)=0 \Leftrightarrow \eta(x-y)=0 \Leftrightarrow x-y=0 \Leftrightarrow x=y
$$

(2) $\quad \rho_{\eta}(x, y)=\eta(x-y)=\eta[(+1)(x-y)]=f(|+1|) \eta(x-y)$

$$
\begin{aligned}
& =f(|-1|) \eta(x-y)=\eta[(-1)(x-y)]=\eta(y-x) \\
& =\rho_{\eta}(y, x)
\end{aligned}
$$

$$
\begin{aligned}
& \rho_{\eta}(x, z)=\eta(x-z)=\eta(x-y+y-z) \\
& \leq \eta(x-y)+\eta(y-z)=\rho_{\eta}(x, y)+\rho_{\eta}(y, z)
\end{aligned}
$$

The next two theorems are based on Proposition 4, page 25, Yosida [8].

Theorem 4.4. Let $\aleph$ be a real or complex linear space and let $\eta$ be an $f$-norm on $\aleph$, and let $g$ be a mapping

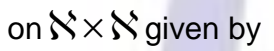

$$
g(x, y)=x+y \text {. }
$$

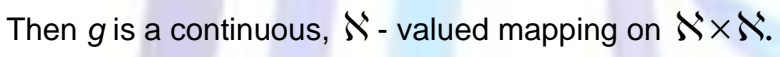

Proof. Given a fixed point $\left(x_{1}, y_{1}\right)$, let $\left(x_{2}, y_{2}\right)$ be a close, variable point. Then

$$
\begin{aligned}
& \rho_{\eta}\left[g\left(x_{1}, y_{1}\right), g\left(x_{2}, y_{2}\right)\right]=\rho_{\eta}\left[\left(x_{1}+y_{1}\right),\left(x_{2}+y_{2}\right)\right] \\
= & \eta\left[\left(x_{1}+y_{1}\right)-\left(x_{2}+y_{2}\right)\right] \leq \eta\left(x_{1}-x_{2}\right)+\eta\left(y_{1}-y_{2}\right)
\end{aligned}
$$

Given $\varepsilon>0$, choose $\delta_{1}, \delta_{2}>0 \ni \delta_{1}<\frac{\varepsilon}{2}$ and $\delta_{2}<\frac{\varepsilon}{2}$.

Then $\eta\left(x_{1}-x_{2}\right)<\delta_{1}$ and $\eta\left(y_{1}-y_{2}\right)<\delta_{2} \Rightarrow \rho_{\eta}\left[g\left(x_{1}, y_{1}\right), g\left(x_{2}, y_{2}\right)\right]<\varepsilon$

Hence $g$ is continuous on $\$$.

Theorem 4.5. If $\aleph$ is an $f$-normed space with $f$-norm $\eta$, then the $\aleph$-valued mapping given by

$$
g[(\alpha, x)]=\alpha x
$$

is continuous.

Proof. $\eta\left[g\left(\alpha_{1}, x_{1}\right)-g\left(\alpha_{2}, x_{2}\right)\right]=\eta\left(\alpha_{1} x_{1}-\alpha_{2} x_{2}\right)=\eta\left(\alpha_{1} x_{1}-\alpha_{2} x_{1}+\alpha_{2} x_{1}-\alpha_{2} x_{2}\right)$

$$
=\eta\left[\left(\alpha_{1}-\alpha_{2}\right) x_{1}+\alpha_{2}\left(x_{1}-x_{2}\right)\right] \leq f\left(\left|\alpha_{1}-\alpha_{2}\right|\right) \eta\left(x_{1}\right)+f\left(\left|\alpha_{2}\right|\right) \eta\left(x_{1}-x_{2}\right)
$$

Given $\alpha_{1}, x_{1}$ and $\varepsilon>0$, choose $\alpha_{2}$ and $x_{2}$, э 


$$
f\left(\left|\alpha_{1}-\alpha_{2}\right|\right)<\frac{\varepsilon}{2 \eta\left(x_{1}\right)} \text { and } \eta\left(x_{1}-x_{2}\right)<\frac{\varepsilon}{2 f\left(\left|\alpha_{2}\right|\right)} .
$$

Then $\quad \eta\left[g\left(\alpha_{1}, x_{1}\right)-g\left(\alpha_{2}, x_{2}\right)\right]<\varepsilon$.

Hence $g$ is continuous.

\section{Examples of $\boldsymbol{f}$-norms}

Example 5.1. The $f$-norm described here is similar to the norm on $L_{p}$ spaces (c.f. Simmons [6], page 215). Let $L^{p}(S)$ be the set of real-valued, continuous functions $g$ defined on an interval $S$ of the real-numbers $\ni|g(x)|^{p}$ is integrable over $S$. Also, let

$$
\left.\eta(g)=\left\{\int_{S} \mid f[g(x)]\right]^{p} d x\right\}^{\frac{1}{p}},
$$

where $f$ satisfies the conditions:

(i) $f(\alpha x)=f(\alpha) f(x), \forall \alpha, x \in \mathfrak{R}$.

(ii) $f$ is a continuous, real-valued function on the non-negative real numbers

(iii) the gradient of $f$ is non-increasing

Then $\eta$ is an $f$-norm, since

(l)

$$
\eta(g)=0 \Rightarrow f[g(x)]=0 \Rightarrow g=0
$$

(II)

$$
\begin{aligned}
& \left.\eta(\lambda g)=\left\{\int_{S} \mid f[\lambda g(x)]\right]^{p} d x\right\}^{\frac{1}{p}}=\left\{\int_{S} \mid f(\lambda) f[g(x)]^{p} d x\right\}^{\frac{1}{p}} \\
& =f(\lambda)\left\{\int_{S} \mid f[g(x)]^{p} d x\right\}^{\frac{1}{p}}=f(\lambda) \eta(g)
\end{aligned}
$$

(III) $\quad \int_{S}|f[g(x)+h(x)]|^{p} d x \leq \int_{S} \mid f\left[g(x)+\left.h(x)\right|^{p-1}|f[g(x)+h(x)]| d x\right.$

$$
\begin{aligned}
& \leq \int_{S}|f[g(x)+h(x)]|^{p-1} \mid f[g(x)]+f[h(x)] d x \\
& =\int_{S} \mid f\left[g(x)+\left.h(x)\right|^{p-1}\left|f[g(x)] d x+\int_{S}\right| f\left[g(x)+\left.h(x)\right|^{p-1} \mid f[h(x)] d x\right.\right. \\
& \leq\left\{\int_{S} \mid f[g(x)+h(x)]^{p^{\prime}(p-1)} d x\right\}^{\frac{1}{p^{\prime}}}\left\{\int_{S}|f[g(x)]|^{p} d x\right\}^{\frac{1}{p}} \\
& +\left\{\int_{S}|f[g(x)+h(x)]|^{p^{\prime}(p-1)} d x\right\}^{\frac{1}{p^{\prime}}}\left\{\int_{S}|f[h(x)]|^{p} d x\right\}^{\frac{1}{p}}
\end{aligned}
$$


from Holder's Inequality, where $p^{\prime}(p-1)=p$

Hence $\left.\quad\left\{\int_{S} \mid f[g(x)+h(x)]^{p} d x\right\}^{\frac{1}{p}} \leq\left\{\int_{S} \mid f[g(x)]\right]^{p} d x\right\}^{\frac{1}{p}}+\left\{\int_{S}|f[h(x)]|^{p} d x\right\}^{\frac{1}{p}}$

That is

$$
\eta(g+h) \leq \eta(g)+\eta(h)
$$

Example 5.2. Let $g$ be a continuous, real-valued function defined on the real numbers, and let the norm of $g$ be given by

$$
\eta(g)=\int_{a}^{b}[|g(x)|]^{\frac{1}{2}} d x, \text { where } 0 \leq a<b
$$

Then

(i)

$$
\begin{aligned}
& \eta(g)=0 \Leftrightarrow g(x)=0 \\
& \eta(\lambda g)=\int_{a}^{b}[|\lambda g(x)|]^{\frac{1}{2}} d x=\lambda^{\frac{1}{2}} \int_{a}^{b}[g(x) \mid]^{\frac{1}{2}} d x=\lambda^{\frac{1}{2}} \eta(g)
\end{aligned}
$$

$$
\begin{gathered}
\eta(g+h)=\int_{a}^{b}[|g(x)+h(x)|]^{\frac{1}{2}} d x \leq \int_{a}^{b}\|g(x)|+| h(x)\|^{\frac{1}{2}} d x \\
\leq \int_{a}^{b}[|g(x)|]^{\frac{1}{2}} d x+\int_{a}^{b}[|h(x)|]^{\frac{1}{2}} d x=\eta(g)+\eta(h)
\end{gathered}
$$

Hence $\eta$ is an $f$-norm, where $f(\lambda)=\lambda^{\frac{1}{2}}$.

The following theorem provides with a way to obtain $f$-norms by choosing the function $f$ in a suitable manner. (c.f. page 215 Simmons [6]).

Theorem 5.3. Let $f$ be a function on the real numbers satisfying the following properties:

(i) $f(\alpha+\beta) \leq f(\alpha)+f(\beta), \forall \alpha, \beta \in \mathfrak{R}$

(ii) $f(1)=1$

(iii) The gradient of $f \geq 0$

(iv) The gradient of $f$ is non-increasing.

(v) $f(|\lambda \alpha|)=f(|\lambda|) f(|\alpha|), \forall \lambda, \alpha \in \mathbb{C}$

Let $\quad \eta_{f p}(\mathbf{x})=\left\{\sum_{i=1}^{n}\left[f\left(\left|\alpha_{i}\right|\right)\right]^{p}\right\}^{\frac{1}{p}}, p>1$, where $\mathbf{x}=\left(\alpha_{1}, \alpha_{2}, \ldots, \alpha_{n}\right) \in \mathbb{C}^{n}$

Then $\eta_{f p}$ is an $f$-norm.

Proof.

(I) $\eta_{f p}(\mathbf{x})=0 \Leftrightarrow f\left(\left|\alpha_{i}\right|\right)=0, i=1,2, \ldots, n . \Leftrightarrow \alpha_{i}=0, i=0,1, \ldots, n \Leftrightarrow \mathbf{x}=\mathbf{0}$

(II) $\eta_{f p}(\lambda \mathbf{x})=\left\{\sum_{i=1}^{n}\left[f\left(\left|\lambda \alpha_{i}\right|\right)\right]^{p}\right\}^{\frac{1}{p}}=\left\{\sum_{i=1}^{n}\left[f(|\lambda|) f\left(\left|\alpha_{i}\right|\right)\right]^{p}\right\}^{\frac{1}{p}}$ 


$$
=f(|\lambda|)\left\{\sum_{i=1}^{n}\left[f\left(\left|\alpha_{i}\right|\right)\right]^{p}\right\}^{\frac{1}{p}}=f(|\lambda|) \eta(\mathbf{x})
$$

(III) Let $\mathbf{y}=\left(\beta_{1}, \beta_{2}, \ldots, \beta_{n}\right)$

$$
\begin{aligned}
& \eta_{f p}(\mathbf{x}+\mathbf{y})=\left\{\sum_{i=1}^{n}\left[f\left(\left|\alpha_{i}+\beta_{i}\right|\right)\right]^{p}\right\}^{\frac{1}{p}} \leq\left\{\sum_{i=1}^{n}\left[f\left(\left|\alpha_{i}\right|+\left|\beta_{i}\right|\right)\right]^{p}\right\}^{\frac{1}{p}} \\
& \quad \leq\left\{\sum_{i=1}^{n}\left[f\left(\left|\alpha_{i}\right|+f\left|\beta_{i}\right|\right)\right]^{p}\right\}^{\frac{1}{p}} \leq\left\{\sum_{i=1}^{n}\left[f\left(\left|\alpha_{i}\right|\right)\right]^{p}\right\}^{\frac{1}{p}}+\left\{\sum_{i=1}^{n}\left[f\left(\left|\beta_{i}\right|\right)\right]^{p}\right\}^{\frac{1}{p}} \\
& \quad \leq \eta_{f p}(\mathbf{x})+\eta_{f p}(\mathbf{y})
\end{aligned}
$$

Hence $\eta_{f p}$ is an $f$-norm. $\square$

\section{Convex Sets}

The next two theorems are based on Proposition 2, page 24, Yosida [8].

Theorem 6.1. Let $\eta$ be an $f$-norm on the space $X$ and let $c$ be any positive number. Then the set $M_{n c}=\{x \in X \mid \eta(x) \leq n c, n \in \Re, n \geq 1\}$ has the following properties:

(i) $0 \in M_{c}$

(ii) $x, y \in M_{c}, 0<\alpha<1 \Rightarrow \alpha x+(1-\alpha) y \in M_{2 c}$

(That is, $M_{c}$ is 2-convex).

(iii) $x, y \in M_{c}, 1<\alpha<k$ and $1<k-\alpha \Rightarrow \alpha x+(k-\alpha) y \in M_{k c}$

(iv) $M_{c}$ is balanced.

(v) $M_{c}$ is absorbing.

\section{Proof.}

(i) $\eta(0)=0 \leq c . \therefore 0 \in M_{c}$.

(ii) $\eta[\alpha x+(1-\alpha) y] \leq \eta(\alpha x)+\eta[(1-\alpha) y]=f(\alpha) \eta(x)+f(1-\alpha) \eta(y)$

$$
\leq[f(\alpha)+f(1-\alpha)] c \leq(1+1) c=2 c
$$

since $f$ is monotone increasing and so $\alpha<1 \Rightarrow f(\alpha) \leq f(1)=1$

and similarly $f(1-\alpha) \leq 1$

(iii) $\quad \eta[(\alpha x)+(k-\alpha) y] \leq \eta(\alpha x)+\eta[(k-\alpha) y]$

$$
\begin{aligned}
& =f(\alpha) \eta(x)+f(k-\alpha) \eta(y) \leq[f(\alpha)+f(k-\alpha)] c \\
& \leq[\alpha+(k-\alpha)] c=k c
\end{aligned}
$$

Therefore $\alpha x+(k-\alpha) y \in M_{k c}$

(iv) $\eta(\alpha x)=f(|\alpha|) \eta(x) \leq \eta(x) \leq c$. 
(v) Given $x \in X$, let $\alpha$ be so large that $f\left(\left|\alpha^{-1}\right|\right) \leq \frac{c}{\eta(x)}$.

Then $\quad \eta\left(\alpha^{-1} x\right) \leq c$

Therefore $\left(\alpha^{-1} x\right) \in M_{c}$.

Theorem 6.2. $\quad \eta(x)=\inf _{\alpha>0, \alpha^{-1} x \in M_{c}} \frac{c}{f\left(\alpha^{-1}\right)}$

Proof. $\left[\alpha^{-1} x \in M_{c}\right] \Leftrightarrow \eta\left(\alpha^{-1} x\right) \leq c \Leftrightarrow f\left(\alpha^{-1}\right) \eta(x) \leq c \Leftrightarrow \eta(x) \leq \frac{c}{f\left(\alpha^{-1}\right)}$

and the result follows.

\section{Limits}

The next three theorems are based on Proposition 1, page 31, Yosida [8]

Theorem 7.1. In a space $\aleph$ with an $f$-norm $\eta$, we have

$$
\rho-\lim _{n \rightarrow \infty} x_{n}=x \Rightarrow \lim _{n \rightarrow \infty} \eta\left(x_{n}\right)=\eta(x)
$$

Proof. Assume that $\rho_{\eta}-\lim _{n \rightarrow \infty} x_{n}=x$.

Given

$$
\varepsilon>0, \exists N \text { э } \rho\left(x_{n}, x\right)<\varepsilon, \forall n>N
$$

$$
\begin{gathered}
\therefore\left|\eta\left(x_{n}\right)-\eta(x)\right| \leq \eta\left(x_{n}-x\right)=\rho_{\eta}\left(x_{n}, x\right)<\varepsilon, \forall n>N . \\
\therefore \lim _{n \rightarrow \infty} \eta\left(x_{n}\right)=\eta(x)
\end{gathered}
$$

Theorem7.2. $\rho_{\eta}-\lim _{n \rightarrow \infty} x_{n}=x$ and $\rho_{\eta}-\lim _{n \rightarrow \infty} y_{n}=y \Rightarrow \rho_{\eta}-\lim _{n \rightarrow \infty}\left(x_{n}+y_{n}\right)=x+y$

Proof. Assume that $\rho_{\eta}-\lim _{n \rightarrow \infty} x_{n}=x$ and $\rho_{\eta}-\lim _{n \rightarrow \infty} y_{n}=y$

Then given $\varepsilon>0, \exists N \ni \eta\left(x_{n}-x\right)<\frac{\varepsilon}{2}$ and $\eta\left(y_{n}-y\right)<\frac{\varepsilon}{2}, \forall n>N$.

$\therefore \eta\left[\left(x_{n}+y_{n}\right)-(x+y)\right]=\eta\left[\left(x_{n}-x\right)+\left(y_{n}-y\right)\right] \leq \eta\left(x_{n}-x\right)+\eta\left(y_{n}-y\right) \leq \varepsilon, \forall n>N$.

$\therefore \rho_{\eta}-\lim _{n \rightarrow \infty}\left(x_{n}+y_{n}\right)=x+y$

Theorem 7.3. $\lim _{n \rightarrow \infty} \alpha_{n}=\alpha$ and $\rho_{\eta}-\lim _{n \rightarrow \infty} x_{n}=x \Rightarrow \rho_{\eta}-\lim _{n \rightarrow \infty} \alpha_{n} x_{n}=\alpha x$, where $\alpha_{n} \in \mathfrak{R}$ and $x_{n} \in \aleph$.

Proof. Assume that $\lim _{n \rightarrow \infty} \alpha_{n}=\alpha$ and $\rho_{\eta}-\lim _{n \rightarrow \infty} x_{n}=x$.

Then $\exists M_{0} \ni\left|\alpha_{n}\right|<M_{0}$ and $\eta(x)<M_{0}, \forall n$.

Since $\left\{\alpha_{n}\right\}$ converges and $f$ is continuous, $\left\{f\left(\alpha_{n} \mid\right)\right\}$ also converges and so is bounded. Hence $\exists M_{1}>0$ э $f\left(\left|\alpha_{n}\right|\right)<M_{1} \forall n$. 
Let

$$
M=\max \left\{M_{0}, M_{1}\right\}
$$

Now

$$
\exists \delta_{1}>0 \text { э }\left|\alpha-\alpha_{n}\right|<\delta_{1} \Rightarrow f\left(\left|\alpha-\alpha_{n}\right|\right)<\frac{\varepsilon}{2 M} \text {. }
$$

Also

$$
\exists N_{1}>0 \text { э } n>N_{1} \Rightarrow\left|\alpha-\alpha_{n}\right|<\delta_{1}
$$

and

$$
\exists N_{2}>0 \text { э } n>N_{2} \Rightarrow \eta\left(x-x_{n}\right)<\frac{\varepsilon}{2 M} .
$$

Hence $\forall n>N=\max \left\{N_{1}, N_{2}\right\}$

$$
\begin{aligned}
\rho_{\eta}\left(\alpha x, \alpha_{n} x_{n}\right) & =\eta\left(\alpha x-\alpha_{n} x_{n}\right) \leq \eta\left(\alpha x-\alpha_{n} x\right)+\eta\left(\alpha_{n} x-\alpha_{n} x_{n}\right) \\
= & \left.f\left(\left|\alpha-\alpha_{n}\right|\right) \eta(x)+f\left(\alpha_{n}\right)\right) \eta\left(x-x_{n}\right) \\
< & \frac{\varepsilon}{2 M} \cdot M+M \cdot \frac{\varepsilon}{2 M}=\varepsilon
\end{aligned}
$$

$\therefore \rho_{\eta}-\lim _{n \rightarrow \infty} \alpha_{n} x_{n}=\alpha x$

\section{REFERENCES}

[1] Hatami, H. 2009 On generalizations of Gower's norm. Doctoral thesis. University of Toronto.

[2] Lee, J. A. and Versleysen M. 2005. Generalization of the Lp-norm for time series and its application to selfgeneralizing maps. $5^{\text {th }}$ workshop on Self-Organizing Maps, Paris, 5-8 September, 740-773.

[3] Matkowski, J. 2013. Subhomogeneity and subadditivity of the Lp-norm like functionals. J. Math. Anal. Appl. 404,172 $-184$.

[4] Ramsay, J. and Silverman, B. 1997. Functional Data Analysis, Springer Series in Statistics, Springer, Berlin.

[5] Roni, F., Delannay, N., Conan-Guez, B. and Verleysen, M. 2005. Representation of functional data in neural networks, Neurocomputing 64, 183-210.

[6] Simmons, G. F. 1963. Introduction to Topology and Modern Analysis, McGraw-Hill Kogakusha Ltd, Tokyo.

[7] Urbanski, R. 1985. A generalization of the Koshi-Shimogaki quasi-norm and a topology in the modular spaces in the sense of Nakano, Arch. Math. 45, 366-373.

[8] Yosida, K. 1980. Functional Analysis, Springer-Verlag, New York.

\section{Author' biography with Photo}

I am from Trinidad, West Indies and studied for the B.Sc., M. Phil. and Ph.D. in Mathematics at the University of the West Indies. After completing the Ph. D. in Combinatorics and Coding Theory, I worked at the University of Zimbabwe, the University of Namibia, the University of Virginia, the University of the West Indies, the College of Science, Technology and Applied Arts of Trinidad and Tobago, and the University of Trinidad and Tobago.

My Master of Philosophy Degree is in Functional Analysis and I continue to be interested in this area; so although I have done research in Combinatorics mainly, at present my research in more in Functional Analysis, particularly Measure Theory. 